\title{
Machine learning methods for anomaly detection in IoT networks, with illustrations
}

\author{
Vassia Bonandrini ${ }^{1}$, Jean-François Bercher ${ }^{2}$ and Nawel Zangar ${ }^{2}$ \\ ${ }^{1}$ ESIEE Paris, France \\ vassia.bonandrinidedu.esiee.fr \\ ${ }^{2}$ LIGM, UMR 8049, École des Ponts, UPEM, ESIEE Paris, CNRS, UPE, France \\ jf.bercherdesiee.fr, nawel.zangardesiee.fr
}

\begin{abstract}
T devices have been the target of 100 million attacks in the first half of 2019 [1]. According to [2], there will be more than 64 billion Internet of Things (IOT) devices by 2025. It is thus crucial to secure loT networks and devices, which include significant devices like medical kit or autonomous car. The problem is complicated by the wide range of possible attacks and their evolution, by the limited computing resources and storage resources available on devices. We begin by introducing the context and a survey of Intrusion Detection System (IDS) for loT networks with a state of the art. So as to test and compare solutions, we consider available public datasets and select the CIDDS-001 Dataset. We implement and test several machine learning algorithms and show that it is relatively easy to obtain reproducible results [20] at the state-of-the-art. Finally, we discuss embedding such algorithms in the loT context and point-out the possible interest of very simple rules.
\end{abstract}

Keywords: Internet of Things, IoT, IDS, NIDS, Intrusion Detection System, rules, CIDDS-001

\section{Introduction}

The problem of securing electronic devices is as old as computers exist, but with time computers have gained more and more resources, so IDS in these devices became more efficient. Now, a lot of different small devices without the power of modern computers are connected to a network and are the target of many attacks. Moreover, every loT system is different and has specific worries depending on the type of the attack (DDoS, Blackhole, Sybil Attack...) they want to be protected from. Wireless Sensor Networks (WSN) for instance has unique characteristics such as limited power supply, low transmission bandwidth, small memory size, and data storage [3]. It is thus crucial to develop and deploy new IDS.

Section 2 presents a brief review of the literature and Section 3 presents the problem of selecting or simulating a dataset to test IDS. Section 4 presents the implementation, tests, and results of several machine learning algorithms for outlier detection for CIDDS-001 dataset. Section 5 presents some simple decision rules that 
can be introduced in an loT network to work like an IDS. Section 6 presents a summary of the conclusions and future work.

\section{Related work}

Doshi et al. [4] simulate loT networks with raspberry Pi and virtual machines. They collect network data from their system and test this data with 5 algorithms: Knearest neighbors, Support Vector Machine (SVM) with linear kernel, decision tree, random forest, and neural network. The specificity of their work is to use stateful features and they get up to $30 \%$ better performance compared to without these features.

Hussain et al. [5] list for each problem many surveys that use machine learning techniques (table IV on the document). For anomaly and intrusion detection:

- $\quad$ K-means clustering and Decision Tree [6]

- $\quad$ Artificial Neural Network ANN [7]

- $\quad$ Novelty and Outlier Detection [8]

- Decision Tree [9]

- $\quad$ Naive Bayes [9], [10]

Butun et al. [3] classify the IDS methodology of IDS in 3 categories:

1 - Anomaly Based detection:

We create an activity profile for each member of the network and a certain amount of deviation is reported as an anomaly. This method is adequate to detect never known attacks but we need to update the profiles periodically because the network behavior can change rapidly.

2 - Misuse based detection

A signature (profile) of the previously known attack is used and is used as a reference to flag the next attacks. The disadvantage of this method is that it cannot detect new type of attacks, but the false positive rate is very low.

3 - Specification based detection

That's a mix of the previous ones, "a set of specifications and constraints that describe the correct operation of a program or protocol is defined." [3] But it takes a lot of time to develop special rules to get a low false-positive rate.

Some surveys have unclear results, sometimes there is no result. There are also very few simulations and implementations in real systems.

\section{Dataset}

Selecting a dataset to design and evaluate NIDS ML-based algorithms is not immediate and may be a full part challenge.

One of the most used datasets is the KDD cup99 set, but it still presents defaults, as emphasized by Tavallaee et al. [11]:

- a lot of redundant measures 
- $\quad$ some parts of the train set were used as test sets in some studies

- $\quad$ set is too long forcing to take only part of the set.

Ring et al. [12] did an exhaustive list of network-based detection data set and compared them. One of the recent and not too heavy dataset is the CIDDS-001 (Coburg Intrusion Detection Data Set) [13] which was described as follows:

"The CIDDS-001 data set was captured within an emulated small business environment in 2017, contains four weeks of unidirectional flow-based network traffic, and comes along with a detailed technical report with additional information. As special feature, the data set encompasses an external server which was attacked in the internet. In contrast to honeypots, this server was also regularly used by the clients from the emulated environment. The CIDDS-001 data set is publicly available and contains SSH brute force, DoS and port scan attacks as well as several attacks captured from the wild." [12]

The dataset contains 14 features as follow.

Table 1. Features within the CIDDS-001 data set, from [13]

\begin{tabular}{|c||l||l||}
\hline \multicolumn{1}{|c|}{ id } & Attribute Name & Attribute Description \\
\hline \hline 1 & Src IP & Source IP Address \\
\hline 2 & Src Port & Source Port \\
\hline 3 & Dest IP & Destination IP Address \\
\hline 4 & Dest Port & Destination Port \\
\hline 5 & Proto & Transport Protocol (e.g. ICMP, TCP, or UDP) \\
\hline 6 & Date first seen & Start time flow first seen \\
\hline 7 & Duration & Duration of the flow \\
\hline 8 & Bytes & Number of transmitted bytes \\
\hline 9 & Packets & Number of transmitted packets \\
\hline 10 & Flags & OR concatenation of all TCP Flags \\
\hline 11 & Class & Class label (Normal, Attacker, Victim) \\
\hline 12 & AttackType & Type of Attack (PortScan, DoS, Bruteforce, PingScan) \\
\hline 13 & AttackID & Unique Attack id \\
\hline 14 & AttackDescription & Additional information about the set attack parameters \\
\hline
\end{tabular}

In our experiment, we used the "Class" attribute as the target for classification, removed the AttackType, AttackID and AttackDescription features which are obviously correlated with the "attacker" class. Furthermore, since IPs were anonymized, they do not convey information so we also removed them. We also use "Date first seen" as the x-axis. Finally, we transformed Flags, Class and Proto, which are categorical features, into "dummy variables" by one-hot encoding.

In the CIDDS-01 dataset, we used the internal-week1 subset of observations, as it contains 42 of the 92 attacks on the entire dataset.

Anomalies are labeled as victim or attacker. However, this file has more than 8 million rows and less than $20 \%$ are anomalies. Hence we face a case of imbalanced classes. In such a case, the more represented class can have a "masking effect" on 
the others; this has been studied in [14] for this dataset. Given the high number of instances available, rebalancing the classes can be simply done by subsampling the majority class (otherwise, one can also oversample the minority classes by creating new, synthetic, instances). In our case, we decided to (a) shuffle the data, (b) keep half of the data for a final evaluation (c) subsample the other half to keep about 180000 instances per class.

\section{$4 \quad$ Experiments and results}

The experiments were carried out using Google Colaboratory with 32GB of ram and the Tensor Processing Unit acceleration material.

The metrics that were used to evaluate the performance of the algorithm include the classification accuracy, precision, recall and F1-score. These metrics are expressed by the equations below,

$$
\begin{aligned}
& \text { Accuracy }=\frac{T P+T N}{T P+T N+F P+F N} \\
& \text { Precision }=\frac{T P}{T P+F P} \\
& \text { Recall }=\frac{T P}{T P+F N} \\
& \text { F1-score }=\frac{2 . \text { Precision } . \text { Recall }}{\text { Precision }+ \text { Recall }}
\end{aligned}
$$

where, TP, TN, FP and FN stand for true positives, true negatives, false positives and false negatives, respectively.

We shuffle the set and we take $33 \%$ of the set as the test set and $66 \%$ as the train set. Then, we classify the traffic with 4 algorithms: K-Nearest Neighbors (KNN), Decision Tree (DT), Random Forest (RF) and Neural Network (NN). We used the python sklearn package to do our test.

For the KNN, we use only 1 neighbour with a uniform weight function. We get a global accuracy of $99.27 \%$; other metrics are reported Table 2. 
Table 2. Results with the K Nearest Neighbour algorithm

\begin{tabular}{|l|l|l|l|}
\hline KNN & precision & recall & f1-score \\
\hline attacker & 0.9633 & 0.9972 & 0.9799 \\
\hline normal & 0.9996 & 0.9916 & 0.9956 \\
\hline victim & 0.9573 & 0.9988 & 0.9976 \\
\hline
\end{tabular}

For the Decision Tree, we used the default parameters, which is the Gini criterion for measuring the quality of a split and maximum expansion of nodes. We already obtained a global accuracy of $99.89 \%$; other performance metrics are given Table 3.

Table 3. results with the Decision Tree algorithm

\begin{tabular}{|l|l|l|l|}
\hline DT & precision & recall & f1-score \\
\hline attacker & 0.9956 & 0.9982 & 0.9969 \\
\hline normal & 0.9998 & 0.9990 & 0.9994 \\
\hline victim & 0.9946 & 0.9996 & 0.9970 \\
\hline
\end{tabular}

For instance, we get something as shown in Fig. 1 for the beginning of the tree:

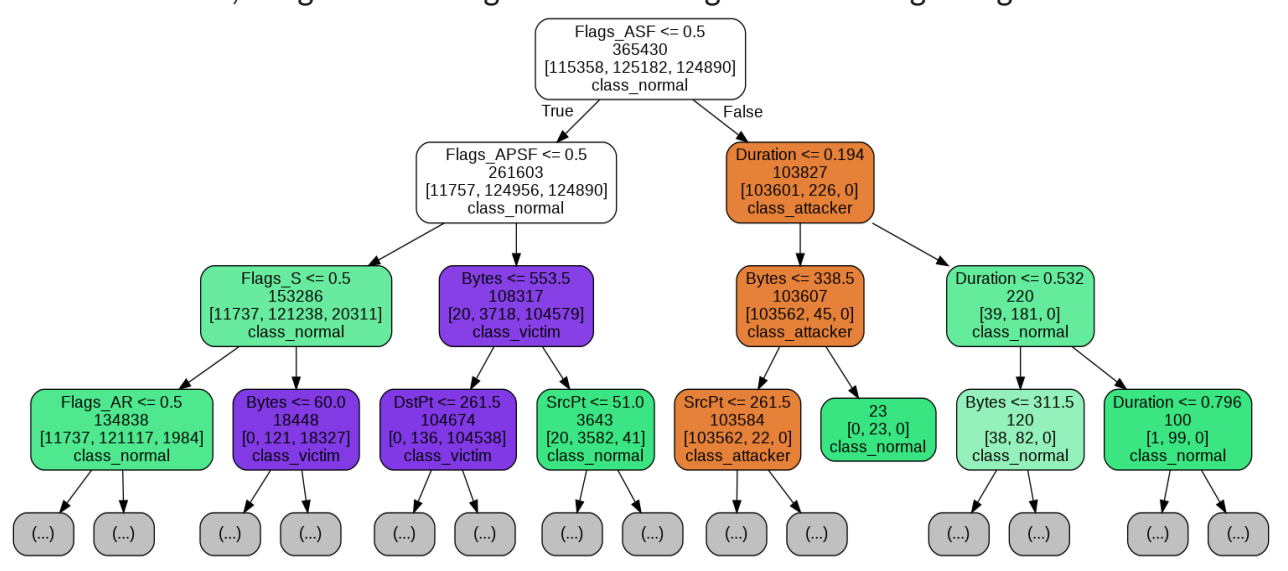

Fig. 1. First nodes of the decision tree 
The decision tree has a depth of 31, a total of 563 nodes and 282 leaves, thus 281 tests. From this tree, it is possible to deduce, and even generate automatically, a classification script (see the source code [20] for an example). Running this code on an instance will predict the classification with $99.88 \%$ accuracy.

For the RF, we selected the best parameters using a grid search strategy, which consists of computing the performance, by cross validation, on a grid of possible parameters values, and then selecting the best estimator. We used global accuracy as the performance metric. In particular, we used 800 trees with a max depth of 20 . We get a global accuracy of $99.95 \%$, with the performances reported in Table 4.

Table 4. results with the Random Forest algorithm

\begin{tabular}{|l|l|l|l|}
\hline RF & precision & recall & f1-score \\
\hline attacker & 0.9982 & 0.9981 & 0.9982 \\
\hline normal & 0.9997 & 0.9996 & 0.9997 \\
\hline victim & 0.9981 & 0.9993 & 0.9997 \\
\hline
\end{tabular}

An interesting outcome of the random forest is that we can extract which are the most important features in computing the classification. The features are shown by importance on Fig. 2. 


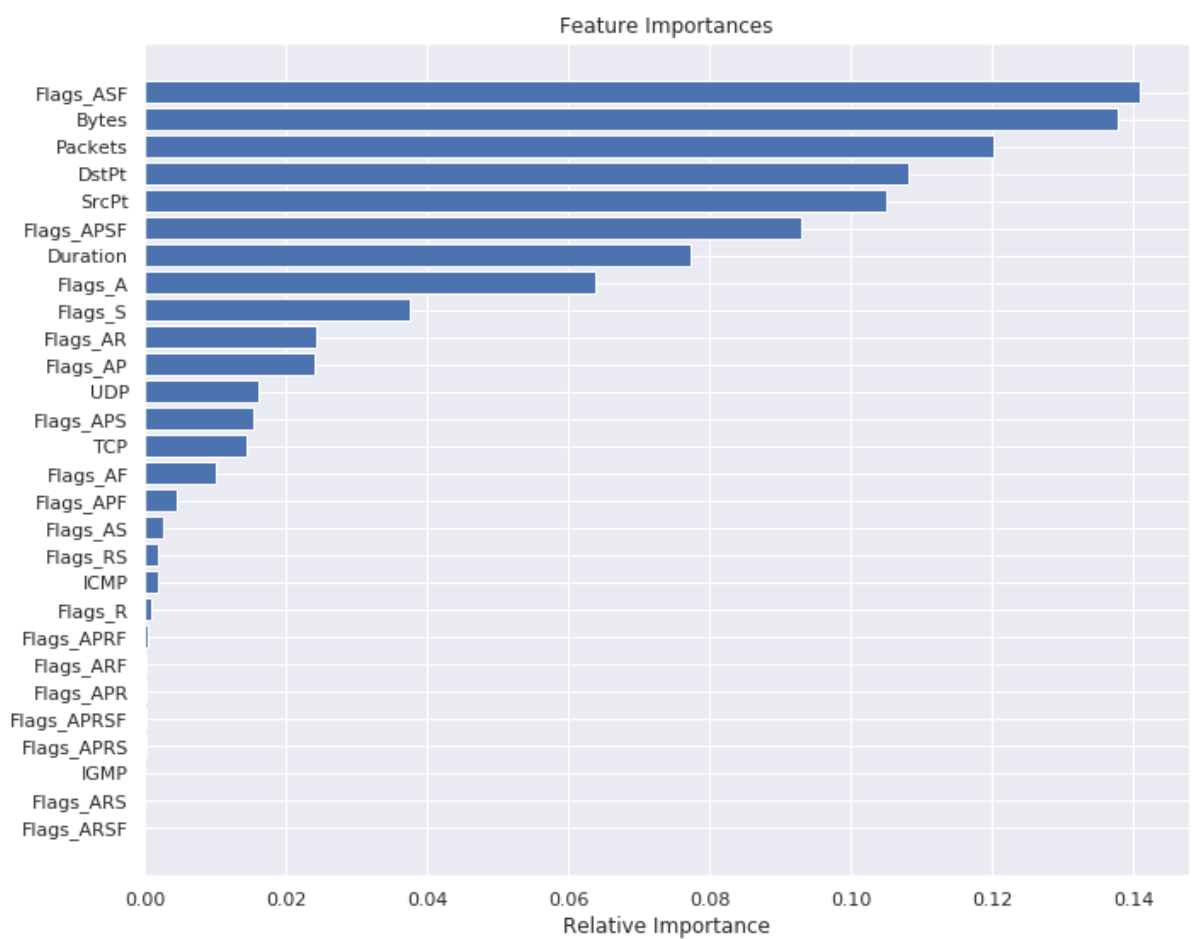

Fig. 2. Relative importance of features in the CIDDS-01 dataset.

For the Neural Network, we used the multi-layer perceptron classifier. This model optimizes the log-loss function using LBFGS or stochastic gradient descent. We used 100 neurons in the hidden layer with the rectified linear unit function for the hidden layer activation function. The maximum iteration was set to 200 . We finally obtained a global accuracy of $99.25 \%$; performances metrics for the different classes are shown Table 5.

Table 5. Results with the Neural Network algorithm

\begin{tabular}{|l|l|l|l|}
\hline NN & precision & recall & f1-score \\
\hline attacker & 0.9929 & 0.9938 & 0.9933 \\
\hline normal & 0.9906 & 0.9962 & 0.9934 \\
\hline victim & 0.9914 & 0.9883 & 0.9899 \\
\hline
\end{tabular}

For the CIDDS-01 dataset and the ML-based algorithms, we obtained very high accuracies. As mentioned in [12], for this particular dataset, balancing the classes or 
not has a very tight influence on the accuracy, which is already so high. Anyway, by a careful selection of the hyperparameters we recover results similar to the best RFWHICD in [14] (where only two classes normal/attacker were considered).

Table 6. Comparison with related work

\begin{tabular}{|l|l|l|}
\hline Survey & Approach & Accuracy (\%) \\
\hline Verma and Ranga [15] & 2NN & 99.60 \\
\hline Verma and Ranga [16] & DT & 99.90 \\
\hline Tama and Rhee [17] & DNN-10-FCV & 99.90 \\
\hline Idhammad et al. [18] & Entropy+RF & 99.54 \\
\hline Abdulhammed et al. [14] & RF-WHICD & 99.99 \\
\hline Proposed KNN & KNN-1 & 99.27 \\
\hline Proposed DT & DT & 99.89 \\
\hline Proposed RF & RF & 99.95 \\
\hline Proposed NN & NN & 99.25 \\
\hline
\end{tabular}

\section{$5 \quad$ Rules}

The most accurate algorithm is the RF with $99.95 \%$ accuracy. However, it may be difficult to embed on an loT and it lacks interpretability. The problem of extracting simple rules from a forest of decision trees has been considered in the machine learning community. The goal was to find a trade-off between the modelization power of random forests and some simple rules interpretable as in a (small) decision tree. The Skope rules Python library [19] enables us to extract such rules from a random forest. In our experiments, we took all the instances to train the model and extract the rules.

For the victim class, the skope rules are:

- $\quad$ Bytes $>100$

- $\quad$ Duration $<=0.03749999962747097$

- $\quad$ Flags $==$ ASF

And for the attacker class, the identified rules are:

- $\quad$ Dst Pt $<=261$

- $\quad$ Duration <= 0.032500000670552254

- $\quad$ Flags $==$ APSF

With these very simple rules, we already get a global accuracy of $86.88 \%$. Furthermore, by a simple inspection of data, we discovered that adding the 
instances flagged with AR (class victim) or S (class attacker) TCP flags enables us to improve the accuracy to $98.45 \%$, with only $0.35 \%$ are miss classifications.

\section{Conclusion}

We have considered the problem of detecting anomalies or intrusions in loT networks. We have first presented the context and reviewed approaches in the literature. We have focused on machine learning-based methods that can learn directly from data and find what are the important features, without resorting to specialized models of the network or specialized signatures. We have then selected a dataset of network activity with several attacks, which is regularly used to develop NIDS and as a benchmark of proposals. Using standard open-source libraries, we have implemented and evaluated several ML-based algorithms, with performances that are at the state-of-the-art. The sources are available and results easily reproducible [20].

Using and implementing such solutions in an loT network requires to consider the possible computational overhead. For a router or network supervisor, we need a network traffic module to capture the incoming network, and the classifiers, once trained by a decision tree or a random forest, can probably be implemented. For the IoT devices themselves, where consumption and computational costs can be more severely constrained, it is possible to use a decision tree classifier (at most 20 comparison tests and a 840 lines program in Python) or even to use the very simple rules ( 3 tests) derived from a random forest, with a $98.5 \%$ accuracy and a low falsepositive rate. Testing these ideas on real devices and real data is the objective of future efforts.

\section{References}

1. "IoT under fire: Kaspersky detects more than 100 million attacks on smart devices in $\mathrm{H} 1$ 2019."Online]_https://www.kaspersky.com/about/press-releases/2019_iot-under-firekaspersky-detects-more-than-100-million-attacks-on-smart-devices-in-h1-2019. last accessed: 29-Oct-2019.

2. P. Newman, "IoT Report: How Internet of Things technology growth is reaching mainstream companies and consumers," Business Insider, 28-Jan-2019.

3. I. Butun, S. D. Morgera, and R. Sankar, "A Survey of Intrusion Detection Systems in Wireless Sensor Networks," IEEE Communications Surveys \& Tutorials, 1 (16), 266-282, (2014).

4. R. Doshi, N. Apthorpe, and N. Feamster, "Machine Learning DDoS Detection for Consumer Internet of Things Devices," In: 2018 IEEE Security and Privacy Workshops (SPW), 29-35 (2018).

5. F. Hussain, R. Hussain, S. A. Hassan, and E. Hossain, "Machine Learning in loT Security: Current Solutions and Future Challenges," arXiv [cs.CR], 14-Mar-2019.

6. P. Shukla, "ML-IDS: A machine learning approach to detect wormhole attacks in Internet 
of Things," In: 2017 Intelligent Systems Conference (IntelliSys), 234-240 (2017)

7. J. Cañedo and A. Skjellum, "Using machine learning to secure loT systems," In: 2016 14th Annual Conference on Privacy, Security and Trust (PST), pp. 219-222 (2016)

8. N. Nesa, T. Ghosh, and I. Banerjee, "Non-parametric sequence-based learning approach for outlier detection in IoT," Future Gener. Comput. Syst. (82), 412-421, (2018).

9. E. Viegas, A. Santin, L. Oliveira, A. França, R. Jasinski, and V. Pedroni, "A reliable and energy-efficient classifier combination scheme for intrusion detection in embedded systems," Comput. Secur., (78), 16-32, (2018).

10. H. H. Pajouh, R. Javidan, R. Khayami, D. Ali, and K.-K. R. Choo, "A two-layer dimension reduction and two-tier classification model for anomaly-based intrusion detection in IoT backbone networks," IEEE Transactions on Emerging Topics in Computing, 2(7), 314 - 323, (2019).

11. M. Tavallaee, E. Bagheri, W. Lu, and A. A. Ghorbani, "A detailed analysis of the KDD CUP 99 data set," In: 2009 IEEE Symposium on Computational Intelligence for Security and Defense Applications, 1-6 (2009).

12. M. Ring, S. Wunderlich, D. Scheuring, D. Landes, and A. Hotho, "A survey of networkbased intrusion detection data sets," Comput. Secur., (86), 147-167 (2019).

13. M. Ring, S. Wunderlich, D. Gruedl, D. Landes, and A. Hotho, "Technical Report CIDDS-001 data set," (2017).

14. R. Abdulhammed, M. Faezipour, A. Abuzneid, and A. AbuMallouh, "Deep and Machine Learning Approaches for Anomaly-Based Intrusion Detection of Imbalanced Network Traffic," IEEE Sensors Letters, 1(3), 1-4, ( 2019).

15. A. Verma and V. Ranga, "On evaluation of network intrusion detection systems: Statistical analysis of CIDDS-001 dataset using machine learning techniques," Pertanika J. Sci. Technol., 3(26), 1307-1322, (2018).

16. A. Verma and V. Ranga, "Statistical analysis of CIDDS-001 dataset for Network Intrusion Detection Systems using Distance-based Machine Learning," Procedia Comput. Sci., (125), 709-716 (2018).

17. B. A. Tama and K.-H. Rhee, "Attack classification analysis of loT network via deep learning approach," Research Briefs on Information \& Communication Technology Evolution (ReBICTE), (3), 1-9 (2017).

18. M. Idhammad, K. Afdel, and M. Belouch, "Detection System of HTTP DDoS Attacks in a Cloud Environment Based on Information Theoretic Entropy and Random Forest," Security and Communication Networks, (2018).

19. https://github.com/scikit-learn-contrib/skope-rules

20. V. Bonandrini et al., https://github.com/VasgoTheTotoroo/IDS_IoT 